\title{
Determination of antioxidative and enzymatic activity in green and red lettuce cultivars affected by microbiological fertilisers and seasons
}

\author{
Milica Stojanović ${ }^{1,2 *}$, Vuk Maksimović ${ }^{3}$, Dragosav Mutavdžić ${ }^{3}$, Ivana Petrović ${ }^{4}$, Zorica Jovanović ${ }^{4}$ \\ Slađana Savić ${ }^{1}$, Jelena Dragišić Maksimović ${ }^{3}$ \\ ${ }^{1}$ Faculty of Biofarming, Megatrend University, Bulevar maršala Tolbuhina 8, 11070 Belgrade, Serbia, ${ }^{2}$ lceberg Salat Centar, Vinogradska 40, \\ 11271 Surčin, Belgrade, Serbia, ${ }^{3}$ Institute for Multidisciplinary Research, University of Belgrade, Kneza Višeslava 1a, 11000 Belgrade, Serbia, \\ ${ }^{4}$ Faculty of Agriculture, University of Belgrade, Nemanjina 6, 11080 Belgrade, Serbia
}

\section{A B S T R A C T}

\begin{abstract}
Lettuce is a worldwide grown leafy vegetable rich in phytochemicals which are essential in human diet. The aim of this study was to examine the effect of genotype, microbiological fertilisers and season on the rosette fresh weight and components of antioxidant activity. Six cultivars (green 'Kiribati', 'Aleppo', 'Aquino' and red 'Murai', 'Carmesi', 'Gaugin') were grown in a greenhouse experiment during three consecutive seasons (autumn, winter and spring) with application of microbiological fertilisers (EM Aktiv, Vital Tricho and their combination). Green cultivars showed higher fresh weight than red in spring and winter. Green cultivar 'Aleppo' showed the highest fresh weight in control during spring. Microbiological fertilisers led to increased fresh weight in autumn. Mainly, red cultivars showed higher quality parameters compared to green (total antioxidant capacity, total phenolic content, total carotenoids and POD activity). Red cultivar 'Carmesi' showed the highest total antioxidant capacity, total carotenoids, and total phenolic content in winter with combination of fertilisers, Vital Tricho, and in control. In spring, 'Carmesi' and 'Gaugin' showed the highest POD activity with Vital Tricho, and combination of fertilisers. The present study suggested that genotype, fertilisers and season jointly influenced quantity and quality parameters with emphasis on Vital Tricho, and/or combination of fertilisers.
\end{abstract}

Keywords: Antioxidative components; Lettuce; Microbiological fertiliser; Rosette fresh weight; Season

\section{INTRODUCTION}

Lettuce (Lactuca sativa, L.) is a leafy vegetable crop from Asteraceae family. A year - round production is possible in temperate climate with lower temperatures and high light intensity which positively affects yield and quality characteristics. In European Union, total production of lettuce and chicory was $2.943 .648 \mathrm{t}$ with the highest amount produced in Spain, Italy and Germany (FAOSTAT 2017).

Lettuce is rich in minerals, dietary fiber, vitamins, phenolics and other health promoting compounds (Mampholo et al., 2016). Compared to cooked vegetables, lettuce supplies more nutrients, since it is consumed as a fresh salad (Kim et al., 2016). Antioxidant compounds such as carotenoids, anthocyanins and phenolics are recognized to have positive effects on human health such as strengthening immune system, protecting skin, preventing cancer, cardiovascular diseases, macular degeneration, reduce accumulation of triglycerides (Ozcan et al., 2014; Cooperstone and Schwartz, 2016). Peroxidases are also in close connection with phenolics as a diverse group of different enzymes that have role in regulation of different metabolic processes, signaling, pathogen defense, formation of the cell wall and various antioxidant properties, mainly by $\mathrm{H}_{2} \mathrm{O}_{2}$-mediated oxidation of phenolic substrates (Flohe and Ursini, 2008). Boo et al. 2011 reported an increase of anthocyanin and polyphenol production as well as an increase in antioxidant enzyme activities at low temperatures in lettuce plants. The same authors suggested that the response was not strict for the apparent cultivar; nevertheless, the anthocyanin and polyphenol content were mostly stimulated by low ambient temperatures, while POD was affected with high day/night temperatures. According to different literature data, green

\footnotetext{
*Corresponding author:

Milica Stojanović, Faculty of Biofarming, Megatrend University, Bulevar maršala Tolbuhina 8, 11070 Belgrade, Serbia, Iceberg Salat Centar, Vinogradska 40, 11271 Surčin, Belgrade, Serbia. Tel: +381642937556. E-mail: mima1384@hotmail.com
} 
cultivars are supposed to have higher rosette fresh weight compared to red, while higher phytochemical parameters (total antioxidant capacity, total phenolic content, total carotenoids and specific POD activity) are expected in red cultivars (Liu et al., 2007; Llorach et al., 2008; Barickman et al., 2018).

Effective microorganisms are different preparations that contain compatible, selected beneficial microorganisms found in natural environment: lactic acid bacteria, yeasts, actinomycetes, photosynthetic bacteria and fungi (Higa and Parr, 1994). These microorganisms can stimulate plant growth through production of different metabolites (hormones, vitamins, enzymes) and enhance nutrient availability and uptake, suppress different plant pathogens, ameliorate soil structure and fertility, decrease negative effect of inorganic fertilisers (Babalola, 2010). Trichoderma spp. is a fungus genus that contains wide range of different opportunistic, cosmopolite species acting as plant symbionts without evoking defense mechanisms in plants. They have an ability to promote plant growth and health through protection from pathogens, stimulating nutrient uptake and availability, indicating positive effect on root branching, and yield, reducing quantity of inorganic fertilisers, and alleviation of stress factors in plants (Molla et al., 2012; Lopez-Bucio et al., 2015).

Application of effective microorganisms is recognized as a part of sustainable strategy in all agricultural systems. There is a necessity to decrease the quantity of inorganic fertilisers and pesticides and minimize their negative impact on the environment with additional emphasis on reducing the costs of production. It is known that excessive use of nitrogen fertilisers results in high levels of nitrate in leafy vegetables, exposing consumers to health risks (Pavlou et al., 2007; Pérez-Urrestarazu et al., 2019). Contemporary agronomic practices, employing different formulations containing organic matter produced from different groups of beneficial microorganisms, can limit nitrate fertiliser usage in vegetables, resulting in product that easily fulfill food quality standards, limit certain contaminants in food and protect human health. Research of Molla et al. (2012) showed that combining inorganic fertilisers with Trichoderma in tomato production can decrease application of inorganic fertilisers for $50 \%$.

Different studies showed beneficial effect of microbiological fertilisers on the yield and quality parameters in different field crops (Zahir et al., 2008; Dubova et al., 2012; Sharma et al., 2012). In particular, recent studies pointed out to their contribution to quality and quantity parameters in lettuce (Dudaš et al., 2016; Kopta et al., 2018; Stojanović et al., 2020). Trichoderma virens strain GV41 showed positive results on the fresh weight and mineral and vitamin content in lettuce and rocket (Fiorentino et al., 2018).
Hence, the aim of this study was to determine and to compare the effects of genotype, microbiological fertilisers and season on the content of different antioxidant components (total antioxidant capacity, total phenolic content, total carotenoids and specific POD activity), and rosette fresh weight in green and red lettuce cultivars.

Since one of the main goals in modern agronomical practice is to enhance the nutritional levels of vegetables by improving nutrient intake and accumulation, we aimed to correlate the effects of different microbiological fertilisers on the rosette fresh weight and the content of health benefit compounds in green and red lettuce cultivars grown in different seasons.

\section{MATERIAL AND METHODS}

\section{Plant material}

Experimentsal setup involved six lettuce Rijk Zwaan cultivars that belong to three different types: oak (green 'Kiribati', red 'Murai'-L. sativa var. crispa), lollo (green 'Aleppo', red 'Carmesi'-L. sativa var. crispa) and multileaf butterhead (green 'Aquino', red 'Gaugin'-L. sativa var. capitata). Lettuce seedlings were grown in peat cubes produced from the substrate Potgrond H (KlasmannDeilmann GmbH, Germany) under controlled glasshouse conditions in the company Grow Rasad, Irig, Serbia.

\section{Microbiological fertilisers}

In the study, two different microbiological fertilisers were examined. EM Aktiv (EMA; Candor, Em tehnologija, Croatia) is a liquid formulation that contains organic matter produced from different groups of beneficial microorganisms isolated from natural environment. Vital Tricho (VT; Candor, Em tehnologija, Croatia) is a powder formulation of both Trichoderma asperellum and Trichoderma viride $\left(5 \times 10^{9} \mathrm{CFU} / \mathrm{ml}\right)$. Third treatment is combination of EM Aktiv and Vital Tricho (EMA+VT).

\section{Experimental design and growth conditions}

Lettuce plants were cultivated during three consecutive crop seasons (autumn October 11, 2016 to December 7, 2016; winter December 27, 2016 to April 5, 2017; spring April 27, 2017 to June 3, 2017) in the company Iceberg Salat Centar, Surčin, Serbia. Experiments were conducted in the greenhouse without additional heating, in a fertile soil, covered with black mulch film. Chemical analysis showed adequate level of macronutrients and humus (nitrogen-0.22\%; phosphorus-58.35 mg/100g; potassium-32.45 mg/100g and humus-5.02\%) and all experiments were performed without using inorganic fertilisers. Trials were set up in a complete block design with 4 treatments (control - without fertilisation (C), EM Aktiv 
(EMA), Vital Tricho (VT) and combination of EM Aktiv and Vital Tricho (EMA+VT)), in 3 replications. Main plots were $2 \times 1 \mathrm{~m}$ included 32 plants, and with $25 \times 25 \mathrm{~cm}$ density. Microbiological fertilisers were applied, via battery sprayer with drift guard, in the soil before transplanting $(150 \mathrm{ml} / 10$ $1 \mathrm{H}_{2} \mathrm{O}$ EMA, $21 \mathrm{~g} / 101 \mathrm{H}_{2} \mathrm{O}$ VT, and $150 \mathrm{ml}+21 \mathrm{~g} / 101$ $\mathrm{H}_{2} \mathrm{O}$ EMA+VT), and 4 times with foliar application during vegetation period $\left(30 \mathrm{ml} / 61 \mathrm{H}_{2} \mathrm{O}\right.$ EMA, $12 \mathrm{~g} / 61 \mathrm{H}_{2} \mathrm{O}$ VT and $30 \mathrm{ml}+12 \mathrm{~g} / 61 \mathrm{H}_{2} \mathrm{O}$ EMA $\left.+\mathrm{VT}\right)$. Regular agriculture practices were performed during growing cycles.

Climatic parameters were measured using RC-4HC Data Logger. Air temperature and air relative humidity were measured for 24 hours during all experiments. Variations between average day,/ night temperature, and air relative humidity are presented in Table 1.

During experiments in all seasons plants were exposed to different day length (https://www.wunderground.com). Photoperiod changed from the date of transplanting to harvest in autumn (11-9 h), winter (9-13 h) and spring (14-15 h).

\section{Data collection and chemical analysis}

Plants were harvested when they achieved commercial maturity and marketable size, in one-time harvest. Rosette (head) fresh weight was measured immediately after the harvest and results are expressed in grams (g). Lettuce tissue was homogenized, and extracted in $80 \%$ ethanol, and centrifuged at $10000 \times g$ for $10 \mathrm{~min}$, providing supernatant was used for further chemical analysis.

Total antioxidant capacity (TAC) was measured according to Miller et al. (1996) with modification of Böhm et al. (2002). For the analysis, we used ethanolic extracts, $5 \mathrm{mM}$ phosphate buffer solution (PBS, $\mathrm{pH} 7.4$ ) and 2,2'-azinobis (3-ethylbenzthiazoline-6-sulphonic acid) as a source of ABTS $\bullet(7 \mathrm{mM})$. Trolox is used to create standard solutions. Absorbance was recorded spectrophotometrically (UV-VIS spectrophotometer, 1166, Lamboled, Inc.USA) at $734 \mathrm{~nm}$. Results of total antioxidant capacity are expressed as ( $\mu \mathrm{mol}$ $\mathrm{TU} / \mathrm{g} \mathrm{FW})$.

Total phenolic content (TPC) was determined spectrophotometrically (Multiskan Spectrum, Thermo Electron Corporation, Vantaa, Finland) using FolinCiocalteu reagent by the method of Singleton and Rossi (1965). Gallic acid was used as a standard to create a calibration curve. Absorbance was read at $724 \mathrm{~nm}$ and results are expressed as microgram of gallic acid equivalents per gram of fresh weight ( $\mu \mathrm{g}$ GA eq/g FW).

Total carotenoids content was measured spectrophotometrically according to Lichtenthaler and

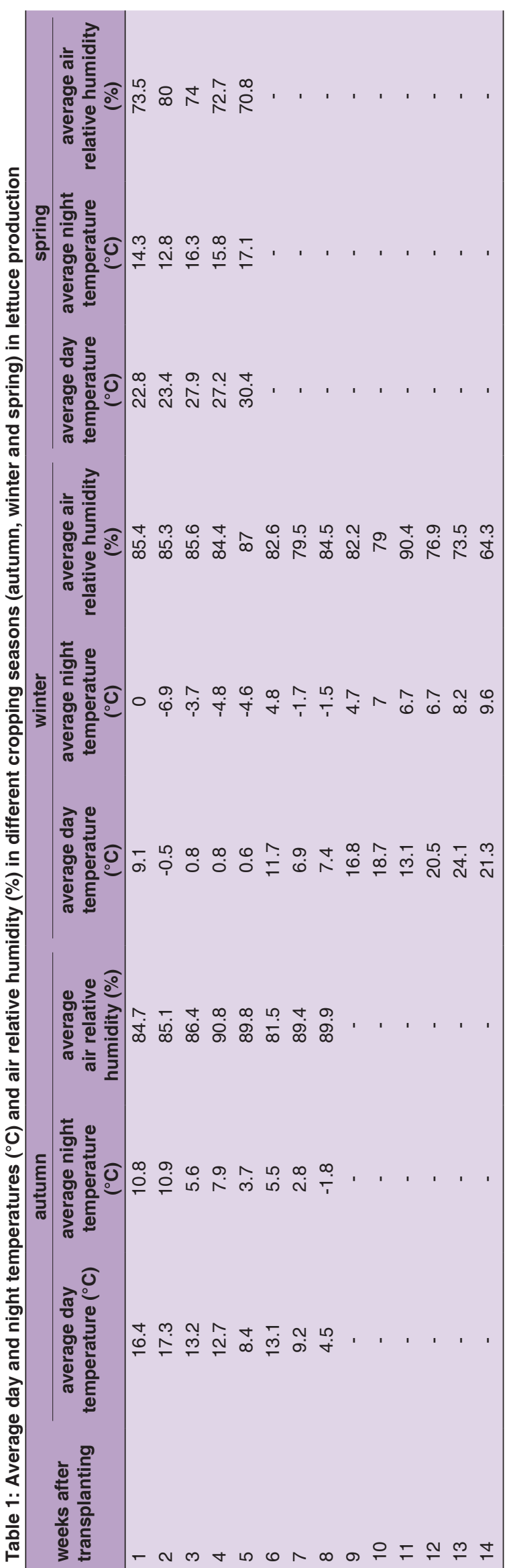


Wellburn (1983). Acetone extracts were centrifuged at $10000 \times g$, at $4{ }^{\circ} \mathrm{C}$ for $10 \mathrm{~min}$. Absorbance was read at $470 \mathrm{~nm}$ and results are expressed as microgram per gram of fresh weight $(\mu \mathrm{g} / \mathrm{g} \mathrm{FW})$.

Total protein content in lettuce leaves was determined by Bradford protein assay (Bradford, 1976). Specific peroxidase (POD, EC 1.11.1.7) activity was measured at $470 \mathrm{~nm}$ through the formation of tetraguaiacol $\left(\varepsilon=25.5 \mathrm{mM}^{-1} \mathrm{~cm}^{-1}\right)$ from guaiacol in the presence of $\mathrm{H}_{2} \mathrm{O}_{2}$. The reaction mixture contained $0.25 \%(\mathrm{v} / \mathrm{v})$ guaiacol and $10 \mathrm{mM} \mathrm{H}_{2} \mathrm{O}_{2}$ in $50 \mathrm{mM}$ sodium phosphate buffer ( $\mathrm{pH}$ 6.0). The unit of specific POD activity was defined as the amount of the enzyme that oxidizes $1 \mu \mathrm{M}$ of substrate per min and expressed per milligram of proteins $(\mathrm{U} / \mathrm{mg}$ prot).

\section{Statistical analysis}

For data analysis we used three-way ANOVA with Tukey's test for post-hoc comparation. We used completely randomized design and all interactions between main factors were statistically significant. For that reason, in post-hoc analysis we compared means of one factor in a way by fixing the levels of the other two factors. All tests were performed at a significance level $\alpha$ of 0.05 . Pearson's correlation was used to test possible correlation between parameters. Statistical analysis was performed using software SPSS Statistics for Windows (Version 22.0. Armonk, NY: IBM Corp) and Microsoft Office Excel 2007 (Microsoft Corp., USA).

\section{RESULTS}

\section{Rosette (head) fresh weight}

Results of rosette (head) fresh weight are presented in Table 2. Rosette (head) fresh weight varied between 58.3$162.0 \mathrm{~g}$ in autumn, 141.0-321.7 $\mathrm{g}$ in winter, and 141.7$347.3 \mathrm{~g}$ in spring. Cultivar 'Aleppo' in spring showed the highest rosette fresh weight $(347.3 \mathrm{~g})$, and the lowest cultivar 'Carmesi' in autumn (58.3 g) in control.

In regard to control treatment, in autumn season, all fertilisers significantly increased rosette fresh weight in cultivars 'Gaugin' and 'Aleppo', while fertiliser EMA+VT had the same effect in cultivars 'Kiribati' and 'Murai', and fertiliser VT in 'Murai'. Opposite to that, in spring, all fertilisers significantly decreased rosette fresh weight in cultivar 'Aleppo', and fertilisers VT and EMA+VT in cultivars 'Murai' and 'Gaugin'. Between treatments, cultivar 'Aleppo' showed the highest rosette fresh weight in winter (321.7 g) with combination of fertilisers. Particularly, season had significant influence on the rosette fresh weight in all cultivars and treatments except in cultivar 'Carmesi' with fertiliser VT.

\section{Total antioxidant capacity (TAC)}

Results of total antioxidant capacity (TAC) measurments are presented in Table 3 . The range of TAC values varied among seasons reaching the highest average values in the winter (254.7-6519.4 $\mu \mathrm{mol} \mathrm{TU} / \mathrm{g}$ ), followed by 376.9$5846.1 \mu \mathrm{mol} \mathrm{TU} / \mathrm{g}$ in autumn, and 192.2-3708.1 $\mu \mathrm{mol}$ TU/g in spring. Cultivar 'Carmesi' showed the highest TAC in winter $(5650.0 \mu \mathrm{mol} \mathrm{TU} / \mathrm{g})$, and the lowest cultivar 'Aquino' in spring $(242.2 \mu \mathrm{mol} \mathrm{TU} / \mathrm{g})$ in control.

In regard to control treatment, in winter, all fertilisers significantly increased TAC in cultivar 'Murai', since in the spring TAC was increased by EMA in 'Gaugin', and $\mathrm{EMA}+\mathrm{VT}$ in 'Aleppo'. All fertilisers significantly decreased TAC in cultivar 'Aleppo' in autumn, and 'Aquino' in winter. Fertilisers EMA and VT significantly decreased TAC too, in cultivars 'Kiribati' (in autumn and spring) and 'Gaugin' (in winter), and EMA+VT in cultivar 'Murai' in autumn. Among treatments, cultivar 'Carmesi' showed the highest TAC $(6519.4 \mu \mathrm{mol} \mathrm{TU} / \mathrm{g})$ in winter with fertiliser EMA+VT. Season affected TAC in all cultivars and treatments, except in cultivars 'Gaugin' (control and EMA+VT) and 'Kiribati' (EMA).

\section{Total phenolic content (TPC)}

Results of estimated total phenolic content (TPC) are presented in Table 4. TPC varied between 175.9-503.0 $\mu \mathrm{g}$ GA eq/g FW in autumn, 18.3-522.7 $\mu \mathrm{g}$ GA eq/g FW in winter, and 88.9-395.5 $\mu \mathrm{g}$ GA eq/g FW in spring. As it was obtained for TAC, cultivar 'Carmesi' in winter showed the highest TPC (522.7 $\mu \mathrm{g}$ GA eq/g), and the lowest cultivar 'Aquino' in spring $(88.9 \mu \mathrm{g}$ GA eq/g) in control.

In regard to control treatment, in winter all fertilisers significantly increased TPC in cultivar 'Murai' since in autumn TPC was increased by VT in cultivar 'Gaugin'. Opposite to that, in winter fertiliser EMA significantly decreased TPC in cultivars 'Gaugin' and 'Carmesi', fertiliser VT in cultivar 'Gaugin', while EMA+VT treatment reduced TPC in cultivar 'Aleppo'. Among treatments, cultivar 'Carmesi' showed the highest TPC (512.9 $\mu \mathrm{g}$ GA eq/g) in winter with fertiliser VT. Season affected TPC in all cultivars and treatments, except in cultivar 'Gaugin' in control.

\section{Total carotenoids}

Results of total carotenoids measurements are presented in Table 5. Total carotenoids content varied between 2.1$5.4 \mu \mathrm{g} / \mathrm{g} \mathrm{FW}$ in autumn, 2.6-9.3 $\mu \mathrm{g} / \mathrm{g} \mathrm{FW}$ in winter, and 2.1-6.6 $\mu \mathrm{g} / \mathrm{g}$ FW in spring. 'Carmesi' in winter showed the highest total carotenoids content $(9.3 \mu \mathrm{g} / \mathrm{g})$, while the lowest was obtained in 'Kiribati' in spring $(2.1 \mu \mathrm{g} / \mathrm{g})$ in control. 
Table 2: Rosette (head) fresh weight ( $\mathrm{g}$ FW) of different lettuce cultivars affected by microbiological fertilisers (C- control; EMA-EM Aktiv; VT- Vital Tricho and EMA+VT- combination of EM Aktiv and Vital Tricho) in different cropping seasons (autumn, winter and spring)

\begin{tabular}{|c|c|c|c|c|c|}
\hline \multirow[t]{2}{*}{ Season } & \multirow[t]{2}{*}{ Cultivar } & \multicolumn{4}{|c|}{ Treatment } \\
\hline & & C & EMA & VT & EMA+VT \\
\hline \multirow[t]{6}{*}{ Autumn } & Kiribati & $88.3 \pm 14.7^{\mathrm{aAx}}$ & $126 \pm 7.6^{\mathrm{cABx}}$ & $146 \pm 20.1^{\mathrm{cABx}}$ & $154.3 \pm 12.9^{\mathrm{cBx}}$ \\
\hline & Murai & $75.7 \pm 12.9^{\mathrm{aAx}}$ & $88 \pm 5.5^{a b A B x}$ & $127 \pm 1^{\mathrm{bcBx}}$ & $124.7 \pm 10.7^{\mathrm{bcBx}}$ \\
\hline & Aquino & $108.3 \pm 15.8^{\mathrm{aAx}}$ & $124.7 \pm 10.7^{\mathrm{bcAx}}$ & $162 \pm 12^{\mathrm{cAx}}$ & $154.7 \pm 7.8^{\mathrm{cAx}}$ \\
\hline & Gaugin & $59.6 \pm 6.2^{a A x}$ & $89 \pm 1^{\mathrm{abcBx}}$ & $91 \pm 2.5^{\mathrm{abBx}}$ & $88.3 \pm 8.4^{\mathrm{abBx}}$ \\
\hline & Aleppo & $70.3 \pm 3.8^{a A x}$ & $120.3 \pm 10.8^{\mathrm{bcBx}}$ & $129.3 \pm 0.7^{\mathrm{bcBCx}}$ & $153 \pm 7.5^{\mathrm{ccx}}$ \\
\hline & Carmesi & $58.3 \pm 4.3^{a A x}$ & $68.7 \pm 7.3^{a A x}$ & $71.7 \pm 3^{\mathrm{aAx}}$ & $74.7 \pm 2^{\mathrm{aAx}}$ \\
\hline \multirow[t]{6}{*}{ Winter } & Kiribati & $257.3 \pm 25.1^{\mathrm{bAy}}$ & $230.3 \pm 27.3^{a A y}$ & $231.3 \pm 32.7^{\text {abAxy }}$ & $275.3 \pm 16.9^{a b A y}$ \\
\hline & Murai & $170.7 \pm 12.5^{\mathrm{aAy}}$ & $199 \pm 31^{\mathrm{aAy}}$ & $217 \pm 13.3^{\mathrm{abAy}}$ & $171.3 \pm 7.7^{\mathrm{aAy}}$ \\
\hline & Aquino & $256.3 \pm 15.5^{\mathrm{bAy}}$ & $277 \pm 27.4^{\mathrm{aAy}}$ & $279.3 \pm 41^{\text {bAy }}$ & $239.3 \pm 12.6^{\mathrm{abAy}}$ \\
\hline & Gaugin & $212.3 \pm 8.1^{\mathrm{abAy}}$ & $222.7 \pm 7.4^{\mathrm{aAy}}$ & $217 \pm 2.5^{\mathrm{abAz}}$ & $199.3 \pm 21.7^{\mathrm{aAy}}$ \\
\hline & Aleppo & $206.3 \pm 13.2^{\mathrm{abAy}}$ & $233 \pm 19.7^{\mathrm{aAy}}$ & $246 \pm 26^{a b A y}$ & $321.7 \pm 40.5^{\mathrm{bAy}}$ \\
\hline & Carmesi & $166 \pm 14.2^{\mathrm{aAy}}$ & $175.7 \pm 13.3^{\mathrm{aAy}}$ & $141 \pm 23.5^{\mathrm{aAx}}$ & $176 \pm 18^{\mathrm{aAy}}$ \\
\hline \multirow[t]{6}{*}{ Spring } & Kiribati & $299.7 \pm 4.3^{\mathrm{bcAz}}$ & $308.7 \pm 28.6^{\mathrm{cAz}}$ & $304.7 \pm 24.2^{\mathrm{cAy}}$ & $293.7 \pm 14.8^{\text {cAy }}$ \\
\hline & Murai & $265 \pm 10^{\mathrm{abBz}}$ & $225.3 \pm 15.5^{\mathrm{abABy}}$ & $203.3 \pm 11.2^{\mathrm{aAy}}$ & $219.7 \pm 1.5^{\mathrm{abABz}}$ \\
\hline & Aquino & $296.3 \pm 15.5^{\text {bcAy }}$ & $286 \pm 11.3^{\text {bcAy }}$ & $281.3 \pm 13.8^{\mathrm{bcAy}}$ & $260.7 \pm 10.1^{\text {bcAy }}$ \\
\hline & Gaugin & $239.3 \pm 22.7^{\mathrm{abBy}}$ & $195 \pm 16.3^{\mathrm{aABy}}$ & $191 \pm 6.2^{\mathrm{aABy}}$ & $169.7 \pm 6.7^{\mathrm{aAy}}$ \\
\hline & Aleppo & $347.3 \pm 23.5^{\mathrm{cBz}}$ & $257.3 \pm 7.9^{a b c A y}$ & $214.7 \pm 9.2^{\mathrm{abAy}}$ & $232 \pm 27.3^{a b c A x y}$ \\
\hline & Carmesi & $212 \pm 19^{\mathrm{aAy}}$ & $189.3 \pm 17.3^{\mathrm{aAy}}$ & $141.7 \pm 20.9^{\mathrm{aAx}}$ & $201 \pm 15.2^{\mathrm{abAy}}$ \\
\hline
\end{tabular}

The data present the means $(n=3) \pm S E$. Values followed by the same letter are not significantly different at the $0.05 \%$ level of probability according to Tuckey's test. The explanations for the symbols are a, b, c - differences between cultivars; A, B, C - differences between treatments; $\mathrm{x}$, y, z - differences between seasons

Table 3: Total antioxidant capacity ( $\mu \mathrm{mol} \mathrm{TU} / \mathrm{g} \mathrm{FW}$ ) of different lettuce cultivars affected by microbiological fertilisers (C- control; EMA-EM Aktiv; VT- Vital Tricho and EMA+VT- combination of EM Aktiv and Vital Tricho) in different cropping seasons (autumn, winter and spring)

\begin{tabular}{|c|c|c|c|c|c|}
\hline \multirow[t]{2}{*}{ Season } & \multirow[t]{2}{*}{ Cultivar } & \multicolumn{4}{|c|}{ Treatment } \\
\hline & & C & EMA & VT & EMA+VT \\
\hline \multirow[t]{6}{*}{ Autumn } & Kiribati & $1434.1 \pm 126.4^{\mathrm{abCy}}$ & $376.9 \pm 52.7^{\mathrm{aAx}}$ & $899.5 \pm 130.5^{\text {aвy }}$ & $1727.6 \pm 59.4^{\mathrm{abCy}}$ \\
\hline & Murai & $1069.2 \pm 157.2^{\mathrm{aBy}}$ & $694.1 \pm 74.1^{\mathrm{aABx}}$ & $886.5 \pm 108.3^{\mathrm{aABx}}$ & $426.8 \pm 21.2^{\mathrm{aAx}}$ \\
\hline & Aquino & $2326.7 \pm 313.1^{\mathrm{bcAy}}$ & $2040.4 \pm 82.1^{\mathrm{bAz}}$ & $2114.9 \pm 279^{\mathrm{aAy}}$ & $1740.4 \pm 186.4^{\text {abAy }}$ \\
\hline & Gaugin & $2935.2 \pm 349.1^{\mathrm{cAx}}$ & $3569.7 \pm 233.1^{\mathrm{cAy}}$ & $5846.1 \pm 963^{\mathrm{bAy}}$ & $5059.3 \pm 803.2^{\mathrm{cAx}}$ \\
\hline & Aleppo & $4688.9 \pm 56.1^{\mathrm{dCz}}$ & $1588.6 \pm 223.4^{\mathrm{bAy}}$ & $1011.4 \pm 10.4^{\mathrm{aAz}}$ & $2811.4 \pm 450.5^{\mathrm{bBy}}$ \\
\hline & Carmesi & $2231.1 \pm 192.4^{\mathrm{bc} A B \mathrm{~B}}$ & $1542.2 \pm 73.8^{\mathrm{bAx}}$ & $2414.4 \pm 269.4^{\mathrm{aABx}}$ & $2778.3 \pm 219.7^{\mathrm{bBx}}$ \\
\hline \multirow[t]{6}{*}{ Winter } & Kiribati & $254.7 \pm 19.3^{\mathrm{aAx}}$ & $285 \pm 9.8^{\mathrm{aAx}}$ & $326.3 \pm 47.1^{\mathrm{aAx}}$ & $316.7 \pm 51.2^{\mathrm{aAx}}$ \\
\hline & Murai & $577 \pm 12.5^{\mathrm{aAx}}$ & $1948 \pm 257.9^{\mathrm{bBy}}$ & $3299.3 \pm 325.1^{\mathrm{bCy}}$ & $3851.7 \pm 303.1^{\mathrm{cCy}}$ \\
\hline & Aquino & $2593.3 \pm 134.7^{\mathrm{bCy}}$ & $829.3 \pm 143.4^{\text {abBy }}$ & $278.3 \pm 6.4^{\mathrm{aAx}}$ & $550.1 \pm 95.3^{\mathrm{aABx}}$ \\
\hline & Gaugin & $2252.2 \pm 80.2^{\mathrm{bBx}}$ & $1007.8 \pm 60.9^{\mathrm{abAx}}$ & $626.1 \pm 33.7^{\mathrm{aAx}}$ & $2776.7 \pm 318.8^{\mathrm{bBx}}$ \\
\hline & Aleppo & $484 \pm 27.6^{\mathrm{aAy}}$ & $588.5 \pm 67.8^{\mathrm{aAx}}$ & $408.9 \pm 20.9^{\mathrm{aAy}}$ & $476.5 \pm 71.5^{\mathrm{aAx}}$ \\
\hline & Carmesi & $5650 \pm 144.3^{\text {сABy }}$ & $5327.8 \pm 554.9^{\text {сАВу }}$ & $5144.4 \pm 16^{\text {сAy }}$ & $6519.4 \pm 184.4^{\mathrm{dBy}}$ \\
\hline \multirow[t]{6}{*}{ Spring } & Kiribati & $577.8 \pm 79^{\mathrm{aBx}}$ & $324.2 \pm 26.5^{\mathrm{aAx}}$ & $327.4 \pm 34^{\mathrm{aAx}}$ & $510.3 \pm 40.9^{\mathrm{aBx}}$ \\
\hline & Murai & $385.6 \pm 52^{\mathrm{aAx}}$ & $571.7 \pm 71.5^{\mathrm{aAx}}$ & $440.9 \pm 61.4^{\mathrm{aAx}}$ & $552.2 \pm 71.2^{\mathrm{aAx}}$ \\
\hline & Aquino & $242.2 \pm 3.2^{\mathrm{aAx}}$ & $266.3 \pm 37^{\mathrm{aAx}}$ & $241.3 \pm 33.3^{\mathrm{aAx}}$ & $222.8 \pm 8.5^{\mathrm{aAx}}$ \\
\hline & Gaugin & $2269.2 \pm 241.4^{\mathrm{bAx}}$ & $3708.1 \pm 28.1^{\mathrm{cBy}}$ & $2446.9 \pm 186.8^{\mathrm{bABx}}$ & $3214 \pm 505.9^{\mathrm{bABx}}$ \\
\hline & Aleppo & $290.8 \pm 10.4^{\mathrm{aAx}}$ & $303.3 \pm 41.5^{\mathrm{aAx}}$ & $192.2 \pm 3.2^{\mathrm{aAx}}$ & $638.1 \pm 53.7^{\mathrm{aBx}}$ \\
\hline & Carmesi & $2142.2 \pm 99.9^{\mathrm{bAx}}$ & $2599.9 \pm 338.5^{\mathrm{bAx}}$ & $2231.9 \pm 139.9^{\mathrm{bAx}}$ & $2788.3 \pm 318.7^{\mathrm{bAx}}$ \\
\hline
\end{tabular}

The data present the means $(n=3) \pm S E$. Values followed by the same letter are not significantly different at the $0.05 \%$ level of probability according to Tuckey's test. The explanations for the symbols are a, b, c, d - differences between cultivars; A, B, C - differences between treatments; $x, y, z$ - differences between seasons

Concerning the control treatment, fertiliser EMA+VT significantly increased total carotenoids content in cultivars 'Aleppo' in winter and 'Murai' in spring whereas fertiliser
VT increased them in cultivar 'Aleppo' in winter. As opposed to that, fertiliser EMA+VT significantly decreased total carotenoids content in cultivars 'Murai' in autumn 
Table 4: Total phenolic content ( $\mu \mathrm{g} \mathrm{GA} \mathrm{eq/g} \mathrm{FW)} \mathrm{of} \mathrm{different} \mathrm{lettuce} \mathrm{cultivars} \mathrm{affected} \mathrm{by} \mathrm{microbiological} \mathrm{fertilisers} \mathrm{(C-} \mathrm{control;}$ EMA-EM Aktiv; VT- Vital Tricho and EMA+VT- combination of EM Aktiv and Vital Tricho) in different cropping seasons (autumn, winter and spring)

\begin{tabular}{|c|c|c|c|c|c|}
\hline \multirow[t]{2}{*}{ Season } & \multirow[t]{2}{*}{ Cultivar } & \multicolumn{4}{|c|}{ Treatment } \\
\hline & & C & EMA & VT & EMA+VT \\
\hline \multirow[t]{6}{*}{ Autumn } & Kiribati & $207.1 \pm 13.4^{\mathrm{aAy}}$ & $175.9 \pm 12.8^{\mathrm{aAy}}$ & $215.4 \pm 5.4^{\mathrm{aAy}}$ & $227.8 \pm 36.7^{\text {aAy }}$ \\
\hline & Murai & $237 \pm 15.9^{\text {abAy }}$ & $213.8 \pm 7.4^{\mathrm{abAy}}$ & $204.9 \pm 20.9^{\mathrm{aAx}}$ & $191.3 \pm 19.1^{\mathrm{aAx}}$ \\
\hline & Aquino & $282.3 \pm 17.2^{\mathrm{abAy}}$ & $252.1 \pm 4.8^{\mathrm{abc} A y}$ & $270.1 \pm 14.8^{\mathrm{aAy}}$ & $229 \pm 15.4^{\mathrm{aAy}}$ \\
\hline & Gaugin & $279.2 \pm 19.2^{\mathrm{abAx}}$ & $367.1 \pm 59.7^{\text {сABy }}$ & $503 \pm 47^{\mathrm{bBy}}$ & $479.3 \pm 47.5^{\mathrm{bABy}}$ \\
\hline & Aleppo & $372.7 \pm 55.7^{\mathrm{bAy}}$ & $313 \pm 24.9^{\mathrm{bcAy}}$ & $226.6 \pm 30.8^{\mathrm{aAy}}$ & $358.5 \pm 59.7^{\text {abAy }}$ \\
\hline & Carmesi & $329.9 \pm 50.8^{\mathrm{abAx}}$ & $204 \pm 14.1^{\mathrm{abAx}}$ & $278.8 \pm 23.1^{\mathrm{aAy}}$ & $385.2 \pm 63.6^{\mathrm{abAxy}}$ \\
\hline \multirow[t]{6}{*}{ Winter } & Kiribati & $123.9 \pm 14.5^{\mathrm{aAx}}$ & $153.5 \pm 6.1^{\text {bcAxy }}$ & $151.6 \pm 26.3^{\mathrm{aAxy}}$ & $130.5 \pm 16.1^{\mathrm{bAxy}}$ \\
\hline & Murai & $174.6 \pm 7.7^{\mathrm{aAx}}$ & $351.1 \pm 1 d^{B z}$ & $458.6 \pm 36.2^{\mathrm{bCy}}$ & $303 \pm 23.7^{\mathrm{cBy}}$ \\
\hline & Aquino & $108.9 \pm 4.8^{\mathrm{aAx}}$ & $104.1 \pm 4^{\mathrm{aAx}}$ & $113.2 \pm 6.4^{\mathrm{aAx}}$ & $115.4 \pm 14.9^{\mathrm{abAx}}$ \\
\hline & Gaugin & $316.8 \pm 7.6^{\mathrm{bBx}}$ & $179.4 \pm 7.3^{\mathrm{cAx}}$ & $164.4 \pm 18.4^{\mathrm{aAx}}$ & $279.5 \pm 31.8^{\mathrm{cBx}}$ \\
\hline & Aleppo & $116.9 \pm 20.1^{\mathrm{aBx}}$ & $123.4 \pm 16.1^{\mathrm{abBx}}$ & $131.1 \pm 9.8^{\mathrm{aBx}}$ & $18.3 \pm 1.1^{\mathrm{aAx}}$ \\
\hline & Carmesi & $522.7 \pm 31.8^{\mathrm{cBy}}$ & $371.5 \pm 8.9^{\mathrm{dAy}}$ & $512.9 \pm 14.3^{\mathrm{bBz}}$ & $476.8 \pm 35.9^{\mathrm{dAB}}$ \\
\hline \multirow[t]{6}{*}{ Spring } & Kiribati & $116.8 \pm 8.6^{\mathrm{aAx}}$ & $122.1 \pm 8.7^{\mathrm{aAx}}$ & $123.9 \pm 18.3^{\mathrm{abAx}}$ & $118.2 \pm 15.8^{\mathrm{aAx}}$ \\
\hline & Murai & $138.4 \pm 16.5^{\mathrm{abAx}}$ & $156.9 \pm 13.4^{\mathrm{aAx}}$ & $136.4 \pm 18.1^{\mathrm{abAx}}$ & $132.2 \pm 17.3^{\mathrm{aAx}}$ \\
\hline & Aquino & $88.9 \pm 7^{\mathrm{aAx}}$ & $98.9 \pm 8.3^{\mathrm{aAx}}$ & $103 \pm 11.1^{\mathrm{abAx}}$ & $103.6 \pm 4.1^{\mathrm{aAx}}$ \\
\hline & Gaugin & $286 \pm 27.7^{\mathrm{cAx}}$ & $340.9 \pm 32.6^{\mathrm{bAxy}}$ & $311 \pm 31.6^{\mathrm{cAx}}$ & $395.5 \pm 37.3^{\text {сAxy }}$ \\
\hline & Aleppo & $92.5 \pm 9.6^{\mathrm{aAx}}$ & $112.4 \pm 3.1^{\mathrm{aAx}}$ & $102.6 \pm 2.3^{\mathrm{aAx}}$ & $115.8 \pm 9.5^{\mathrm{aAx}}$ \\
\hline & Carmesi & $201.4 \pm 4.7^{\mathrm{bAx}}$ & $264.9 \pm 19.1^{\mathrm{bAx}}$ & $186.7 \pm 9.2^{\mathrm{bAx}}$ & $240.3 \pm 32.3^{\mathrm{bAx}}$ \\
\hline
\end{tabular}

The data present the means $(n=3) \pm$ SE. Values followed by the same letter are not significantly different at the $0.05 \%$ level of probability according to Tuckey's test. The explanations for the symbols are a, b, c, d - differences between cultivars; A, B, C - differences between treatments; $x, y, z$ - differences between seasons

Table 5: Total carotenoids ( $\mu \mathrm{g} / \mathrm{g}$ FW) of different lettuce cultivars affected by microbiological fertilisers (C- control; EMA-EM Aktiv; VT- Vital Tricho and EMA+VT- combination of EM Aktiv and Vital Tricho) in different cropping seasons (autumn, winter and spring)

\begin{tabular}{|c|c|c|c|c|c|}
\hline \multirow[t]{2}{*}{ Season } & \multirow[t]{2}{*}{ Cultivar } & \multicolumn{4}{|c|}{ Treatment } \\
\hline & & C & EMA & VT & $\mathrm{EMA+VT}$ \\
\hline \multirow[t]{6}{*}{ Autumn } & Kiribati & $3.5 \pm 0.6^{\mathrm{abAx}}$ & $2.8 \pm 0.3^{\mathrm{abAx}}$ & $2.3 \pm 0.1^{\mathrm{aAx}}$ & $2.5 \pm 0.3^{\mathrm{aAx}}$ \\
\hline & Murai & $5.4 \pm 0.5^{\mathrm{bBy}}$ & $3.8 \pm 0.6^{\mathrm{abABx}}$ & $2.6 \pm 0.2^{\mathrm{abAx}}$ & $2.7 \pm 0.3^{\mathrm{aAx}}$ \\
\hline & Aquino & $2.6 \pm 0.1^{\mathrm{aAx}}$ & $2.9 \pm 0.2^{\mathrm{abAx}}$ & $2.4 \pm 0.003^{\mathrm{aAx}}$ & $2.7 \pm 0.2^{\mathrm{aAx}}$ \\
\hline & Gaugin & $4.4 \pm 0.6^{\mathrm{abAx}}$ & $3.5 \pm 0.3^{\mathrm{abAx}}$ & $5.1 \pm 0.4^{\mathrm{cAx}}$ & $4.3 \pm 0.4^{\mathrm{bAx}}$ \\
\hline & Aleppo & $3.6 \pm 0.1^{\mathrm{abBx}}$ & $2.5 \pm 0.3^{\mathrm{aAx}}$ & $2.1 \pm 0.3^{\mathrm{aAx}}$ & $2.8 \pm 0.3^{\mathrm{aABx}}$ \\
\hline & Carmesi & $5.4 \pm 0.7^{\mathrm{bAx}}$ & $4.4 \pm 0.1^{\mathrm{bAx}}$ & $3.8 \pm 0.4^{\mathrm{bAx}}$ & $4.4 \pm 0.2^{\mathrm{bAx}}$ \\
\hline \multirow[t]{6}{*}{ Winter } & Kiribati & $2.8 \pm 0.4^{\mathrm{aAx}}$ & $3.0 \pm 0.1^{\mathrm{aAx}}$ & $2.6 \pm 0.1^{\mathrm{aAx}}$ & $3.0 \pm 0.1^{\mathrm{aAx}}$ \\
\hline & Murai & $4.3 \pm 0.2^{\mathrm{bAxy}}$ & $5.2 \pm 0.6^{\mathrm{bAx}}$ & $5.5 \pm 0.3^{\mathrm{cAy}}$ & $4.7 \pm 0.6^{\mathrm{abAy}}$ \\
\hline & Aquino & $3.2 \pm 0.1^{\mathrm{aAy}}$ & $3.4 \pm 0.2^{\mathrm{abAx}}$ & $3.5 \pm 0.3^{\text {abAy }}$ & $3.2 \pm 0.3^{\mathrm{aAx}}$ \\
\hline & Gaugin & $4.7 \pm 0.2^{\mathrm{bAx}}$ & $5.4 \pm 0.7^{\mathrm{bAx}}$ & $4.9 \pm 0.3^{\mathrm{bc} A \mathrm{x}}$ & $6.5 \pm 0.7^{\mathrm{bcAx}}$ \\
\hline & Aleppo & $3.2 \pm 0.3^{\mathrm{aAx}}$ & $3.8 \pm 0.05^{\mathrm{abABy}}$ & $4.8 \pm 0.3^{\mathrm{bcBy}}$ & $4.6 \pm 0.2^{\mathrm{abBy}}$ \\
\hline & Carmesi & $9.3 \pm 0.2^{\mathrm{cBy}}$ & $8.6 \pm 0.5^{\mathrm{cABy}}$ & $9.3 \pm 0.5^{\mathrm{dBy}}$ & $7.2 \pm 0.2^{\mathrm{cAy}}$ \\
\hline \multirow[t]{6}{*}{ Spring } & Kiribati & $2.1 \pm 0.3^{\mathrm{aAx}}$ & $2.3 \pm 0.3^{\mathrm{aAx}}$ & $3.0 \pm 0.3^{\mathrm{aAx}}$ & $2.5 \pm 0.3^{\mathrm{aAx}}$ \\
\hline & Murai & $3.5 \pm 0.1^{\mathrm{abcAx}}$ & $4.3 \pm 0.4^{\mathrm{abABx}}$ & $4.4 \pm 0.2^{\mathrm{abABy}}$ & $4.8 \pm 0.1^{\mathrm{bcBy}}$ \\
\hline & Aquino & $3.0 \pm 0.1^{\mathrm{abAxy}}$ & $3.5 \pm 0.4^{\mathrm{abAx}}$ & $2.9 \pm 0.3^{\text {axxy }}$ & $3.7 \pm 0.3^{\mathrm{abAx}}$ \\
\hline & Gaugin & $4.3 \pm 0.7^{\mathrm{bcAx}}$ & $5.5 \pm 0.6^{\mathrm{bAx}}$ & $5.2 \pm 0.3^{\mathrm{bAx}}$ & $5.4 \pm 0.3^{\operatorname{cdAx}}$ \\
\hline & Aleppo & $4.2 \pm 0.4^{\mathrm{bcAx}}$ & $3.9 \pm 0.2^{\mathrm{abAy}}$ & $3.7 \pm 0.2^{\mathrm{abAy}}$ & $3.9 \pm 0.2^{\mathrm{abcAy}}$ \\
\hline & Carmesi & $5.0 \pm 0.4^{\mathrm{cAx}}$ & $5.1 \pm 0.6^{\mathrm{bAx}}$ & $5.2 \pm 0.6^{\mathrm{bAx}}$ & $6.6 \pm 0.5^{\mathrm{dAy}}$ \\
\hline
\end{tabular}

The data present the means $(n=3) \pm S E$. Values followed by the same letter are not significantly different at the $0.05 \%$ level of probability according to Tuckey's test. The explanations for the symbols are $a, b, c, d$ - differences between cultivars; A, B - differences between treatments; $x$, $y$ - differences between seasons

and 'Carmesi' in winter, as well as fertiliser VT in cultivars 'Murai' and 'Aleppo' in autumn and EMA in cultivar
'Aleppo' in autumn. Among treatments, cultivar 'Carmesi' showed the highest total carotenoids content $(9.3 \mu \mathrm{g} / \mathrm{g})$ in 
winter with fertiliser VT. Season affected total carotenoids content in all cultivars and treatments, with the following exceptions: 'Kiribati', 'Gaugin' and 'Aleppo' in control; 'Kiribati', 'Murai', 'Aquino' and 'Gaugin' in EMA; 'Kiribati' and 'Gaugin' in VT; 'Kiribati', 'Aquino' and 'Gaugin' in $\mathrm{EMA}+\mathrm{VT}$.

\section{Peroxidase specific activity (POD)}

Results of peroxidase (POD) specific activity estimations are presented in Table 6 . POD specific activity values varied between $0.02-0.28 \mathrm{U} / \mathrm{mg}$ prot in autumn, $0.004-0.12 \mathrm{U} / \mathrm{mg}$ prot in winter, and $0.02-0.43 \mathrm{U} / \mathrm{mg}$ prot in spring. Cultivar 'Gaugin' in spring showed the highest POD activity $(0.22 \mathrm{U} / \mathrm{mg}$ prot$)$, and the lowest cultivar 'Murai' in winter $(0.004 \mathrm{U} / \mathrm{mg}$ prot $)$ in control.

Regarding to control treatment, in autumn different fertilisers significantly increased POD activity in following manner: EMA and V'T in cultivar 'Carmesi', and EMA+VT in 'Kiribati' and 'Gaugin'. Also, in winter fertiliser EMA significantly improved POD activity in 'Kiribati', VT in 'Carmesi', EMA+VT in 'Gaugin' and 'Aleppo', and all fertilisers in 'Murai'. In spring, POD activity was higher with EMA in 'Kiribati', with VT in 'Murai' and 'Carmesi' and with EMA+VT in 'Gaugin' and 'Carmesi'. Contrary to that, fertiliser EMA and VT significantly decreased POD activity in cultivars 'Gaugin' (spring) and 'Aleppo' (autumn). Among treatments, cultivars 'Carmesi' and
'Gaugin' showed the highest POD activity $(0.43 \mathrm{U} / \mathrm{mg}$ prot) in spring with fertiliser EMA+VT. Season affected POD activity in all cultivars and treatments, with the exception of cultivar 'Gaugin' with EMA, and cultivar 'Murai' with VT.

Results of three-way ANOVA showed significant impact of the genotype, microbiological fertilisers, and season on tested parameters with the exception of the effect of fertilisers, and interaction of genotype and fertiliser, on the rosette fresh weight and total carotenoids content (Table 7). Regardless to that, for all tested parameters interactions between main factors were statistically significant.

\section{Correlations between tested parameters}

Results of rosette fresh weight, TAC, TPC, total carotenoids and POD activity were used to determine possible correlation (Fig.3). Numerous linear correlations were found between tested parameters with emphasize on positive correlation between TAC and TPC $\left(\mathrm{r}=0.91^{* *}\right)$, TAC and total carotenoids ( $\left.\mathrm{r}=0.64^{* *}\right)$, TPC and total carotenoids content $\left(\mathrm{r}=0.56^{* *}\right)$, and TPC and POD activity $\left(\mathrm{r}=0.33^{*}\right)$. On contrary, moderate negative correlation was found between lettuce quantity and quality parameters, such as rosette fresh weight and TPC $\left(r=-0.62^{* *}\right)$, and rosette fresh weight and TAC $\left(r=-0.50^{* *}\right)$.

Table 6: Peroxidase specific activity (U/mg prot) of different lettuce cultivars affected by microbiological fertilisers (C- control; EMA-EM Aktiv; VT- Vital Tricho and EMA+VT- combination of EM Aktiv and Vital Tricho) in different cropping seasons (autumn, winter and spring)

\begin{tabular}{|c|c|c|c|c|c|}
\hline \multirow[t]{2}{*}{ Season } & \multirow[t]{2}{*}{ Cultivar } & \multicolumn{4}{|c|}{ Treatment } \\
\hline & & C & EMA & VT & EMA+VT \\
\hline \multirow[t]{6}{*}{ Autumn } & Kiribati & $0.07 \pm 0.002^{\mathrm{bABz}}$ & $0.08 \pm 0.011^{\mathrm{bBy}}$ & $0.04 \pm 0.003^{\text {abAy }}$ & $0.15 \pm 0.011^{\mathrm{bCz}}$ \\
\hline & Murai & $0.03 \pm 0.003^{\mathrm{aAB} y}$ & $0.02 \pm 0 a^{A x}$ & $0.04 \pm 0.005^{\mathrm{abAx}}$ & $0.02 \pm 0.001^{\mathrm{aAx}}$ \\
\hline & Aquino & $0.06 \pm 0.001^{\text {abABy }}$ & $0.06 \pm 0.006^{\mathrm{abABy}}$ & $0.08 \pm 0.004^{\mathrm{abcBy}}$ & $0.05 \pm 0.005^{\mathrm{aAxy}}$ \\
\hline & Gaugin & $0.06 \pm 0.007^{\mathrm{abAx}}$ & $0.09 \pm 0.009^{\mathrm{bAx}}$ & $0.13 \pm 0.020 \mathrm{~d}^{\mathrm{Ax}}$ & $0.28 \pm 0.024^{\mathrm{cBy}}$ \\
\hline & Aleppo & $0.14 \pm 0.014^{\mathrm{cBy}}$ & $0.1 \pm 0.013^{\mathrm{bABy}}$ & $0.08 \pm 0.010^{\text {bc } A y}$ & $0.11 \pm 0.002^{\mathrm{bABy}}$ \\
\hline & Carmesi & $0.05 \pm 0.007^{\mathrm{abAx}}$ & $0.09 \pm 0.013^{\mathrm{bBx}}$ & $0.09 \pm 0.003^{\operatorname{cdB} x}$ & $0.05 \pm 0.001^{\mathrm{aAx}}$ \\
\hline \multirow[t]{6}{*}{ Winter } & Kiribati & $0.01 \pm 0.001^{\mathrm{aAx}}$ & $0.04 \pm 0.005^{\mathrm{bBx}}$ & $0.01 \pm 0.002^{\mathrm{abAx}}$ & $0.01 \pm 0.001^{\mathrm{aAx}}$ \\
\hline & Murai & $0.004 \pm 0^{\mathrm{aAx}}$ & $0.04 \pm 0.002^{\mathrm{bBy}}$ & $0.04 \pm 0.007^{\mathrm{bcBx}}$ & $0.05 \pm 0.005^{\mathrm{cBy}}$ \\
\hline & Aquino & $0.02 \pm 0.003^{\mathrm{aAB} x}$ & $0.03 \pm 0.005^{\mathrm{abBx}}$ & $0.01 \pm 0.001^{\mathrm{aAx}}$ & $0.03 \pm 0.003^{\mathrm{abcBx}}$ \\
\hline & Gaugin & $0.06 \pm 0.007^{\mathrm{bAx}}$ & $0.09 \pm 0.008^{\mathrm{dABx}}$ & $0.06 \pm 0.006^{\mathrm{cAx}}$ & $0.12 \pm 0.012^{\mathrm{dBx}}$ \\
\hline & Aleppo & $0.01 \pm 0.001^{\mathrm{aAx}}$ & $0.01 \pm 0.002^{\mathrm{aAx}}$ & $0.01 \pm 0 \mathrm{a}^{\mathrm{Ax}}$ & $0.02 \pm 0.002^{\mathrm{abBx}}$ \\
\hline & Carmesi & $0.05 \pm 0.008^{\mathrm{bAx}}$ & $0.07 \pm 0.001^{\mathrm{ABB}}$ & $0.1 \pm 0.015^{\mathrm{dBx}}$ & $0.05 \pm 0^{\mathrm{bc} A x}$ \\
\hline \multirow[t]{6}{*}{ Spring } & Kiribati & $0.04 \pm 0.007^{\mathrm{aAy}}$ & $0.07 \pm 0.006^{\mathrm{aBxy}}$ & $0.05 \pm 0.007^{\text {aABy }}$ & $0.07 \pm 0.005 \mathrm{a}^{\mathrm{ABy}}$ \\
\hline & Murai & $0.02 \pm 0.002^{\mathrm{aAy}}$ & $0.04 \pm 0.005^{\mathrm{aAB} y}$ & $0.05 \pm 0.004^{\mathrm{aBx}}$ & $0.04 \pm 0.006^{\text {авву }}$ \\
\hline & Aquino & $0.05 \pm 0.002^{\mathrm{aAy}}$ & $0.06 \pm 0.005^{\mathrm{aAy}}$ & $0.06 \pm 0.008^{\mathrm{aAy}}$ & $0.05 \pm 0.008^{\mathrm{aAy}}$ \\
\hline & Gaugin & $0.22 \pm 0.006^{\mathrm{bBy}}$ & $0.12 \pm 0.011^{\mathrm{aAx}}$ & $0.31 \pm 0.040^{\mathrm{bBy}}$ & $0.43 \pm 0.012^{\mathrm{bCz}}$ \\
\hline & Aleppo & $0.03 \pm 0.003^{\mathrm{aAx}}$ & $0.04 \pm 0.006^{\mathrm{aAx}}$ & $0.03 \pm 0.002^{\mathrm{aAx}}$ & $0.02 \pm 0.003^{\mathrm{aAx}}$ \\
\hline & Carmesi & $0.19 \pm 0.019^{\mathrm{bAy}}$ & $0.24 \pm 0.042^{\mathrm{bAy}}$ & $0.37 \pm 0.025^{\mathrm{bBy}}$ & $0.43 \pm 0.024^{\mathrm{bBy}}$ \\
\hline
\end{tabular}

The data present the means $(n=3) \pm S E$. Values followed by the same letter are not significantly different at the $0.05 \%$ level of probability according to Tuckey's test. The explanations for the symbols are a, b, c, d - differences between cultivars; A, B, C - differences between treatments; $\mathrm{x}$, $\mathrm{y}$, $\mathrm{z}$ - differences between season 
Table 7: Three-way ANOVA of fresh weight, total antioxidant capacity, total phenolic content, total carotenoids and POD activity as affected by genotype, microbiological fertilisers and season

\begin{tabular}{|c|c|c|c|c|c|}
\hline & $\begin{array}{l}\text { Rosette fresh } \\
\text { weight (g FW) }\end{array}$ & $\begin{array}{c}\text { Total antioxidant capacity } \\
(\mu \mathrm{mol} \mathrm{TU} / \mathrm{g} \text { FW })\end{array}$ & $\begin{array}{l}\text { Total phenolic content } \\
(\mu \mathrm{g} \mathrm{GA} \text { eq/g FW) }\end{array}$ & $\begin{array}{c}\text { Total } \\
\text { carotenoids } \\
(\mu \mathrm{g} / \mathrm{g} \mathrm{FW})\end{array}$ & $\begin{array}{l}\text { POD activity } \\
\text { (U/mg prot) }\end{array}$ \\
\hline Genotype (G) & $* * *$ & $* * *$ & $* * *$ & $* * *$ & $* * *$ \\
\hline Microbiological fertilisers (MF) & NS & $* * *$ & * & NS & $* * *$ \\
\hline Season (S) & $* * *$ & $* * *$ & $* * *$ & $* * *$ & $* * *$ \\
\hline$G \times M F$ & NS & $* * *$ & $* * *$ & NS & $* * *$ \\
\hline$G \times S$ & $* * *$ & $* * *$ & $* * *$ & $* * *$ & $* * *$ \\
\hline MF $\times S$ & $* * *$ & $* * *$ & $* * *$ & $* * *$ & $* * *$ \\
\hline$G \times M F \times S$ & $* *$ & $* * *$ & $* * *$ & $* * *$ & $* * *$ \\
\hline
\end{tabular}

Asterisks indicate significant differences at $* P \leq 0.05 ; * * P \leq 0.01 ; * * * P \leq 0.001$; NS, non-significant

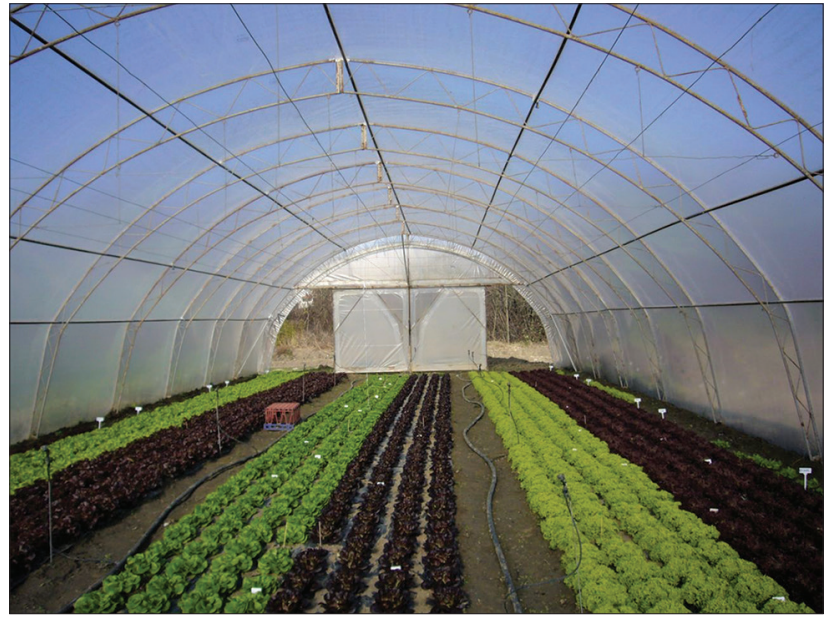

AQ1 Fig 1. Green and red lettuce cultivars grown in the greenhouse with application of four different treatments during autumn

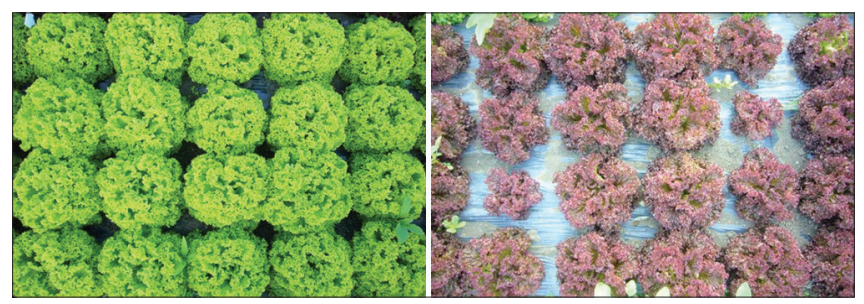

AQ1 Fig 2. Differences in the rosette weight in cultivars 'Aleppo' as the most productive in spring (left) and 'Carmesi' the least productive in autumn (right) in control treatment

\section{DISCUSSION}

Present study indicated that mostly green cultivars showed higher rosette (head) fresh weight, compared to red within same type. Similar results were observed in green and red oak, and romaine lettuce, respectively (Koudela and Petrikova, 2008; Barickman et al., 2018). The highest rosette fresh weight, irrespective to treatment, was obtained with cultivar 'Aleppo', usually followed by cultivar 'Kiribati', and the lowest fresh weight was observed in 'Carmesi' both in control and with application of fertilisers.

Microorganisms can produce hormones, vitamins, enzymes, and other plant stimulating compounds, and they

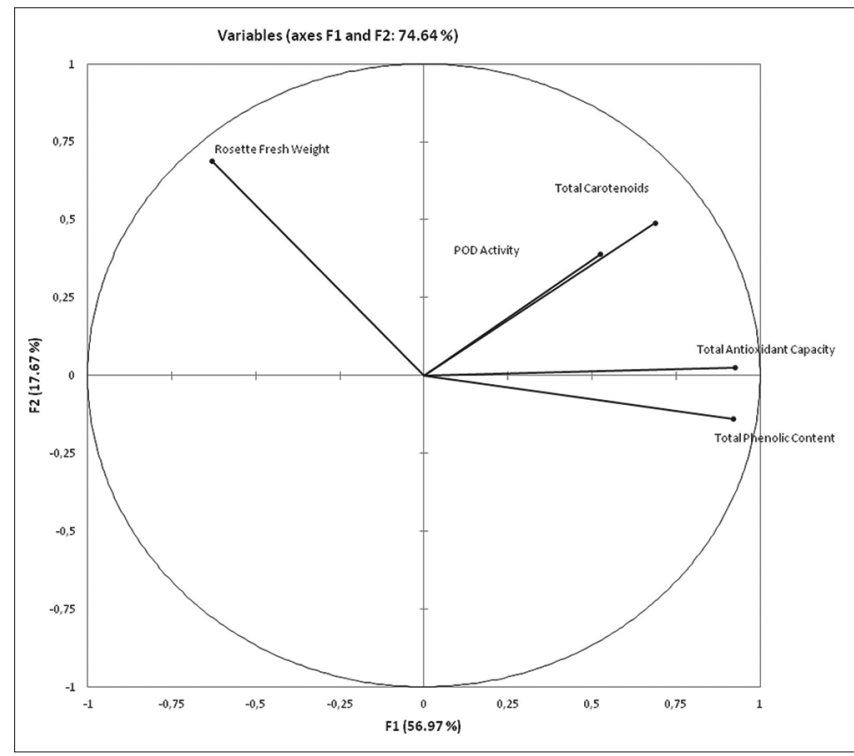

Fig 3. Graph of loadings obtained from all parameters in the study (rosette fresh weight, total antioxidant capacity, total phenolic content, total carotenoids and POD activity)

can stimulate plant growth and yield through increased nutrient availability and uptake. Experiments with effective microorganisms and Trichoderma emphasized their positive effect on yield and fresh weight of different agricultural crops, such as strawberry, tomato, and lettuce (Kowalska, 2011; Molla et al., 2012; 'Tošić et al., 2016). On the contrary, results of Priyadi et al. (2005) showed no positive effect on corn yield with application of effective microorganisms. The impact of effective microorganisms may depend on plant species, soil type, fertility and frequency of use. Effects of Trichoderma preparations depend on cultivar (genotype), ability of Trichoderma strain to colonize root, and the method of application. In this study, cultivars 'Aquino' and 'Carmesi' didn't show statistically significant increase in rosette fresh weight with application of microbiological fertilisers in all seasons, which may be justified as particular interaction between genotype driven adaptations e.g. root exudates, beneficial microorganisms, and Trichoderma, or insufficient quantity of fertilisers. Results of Bal and Altintas (2006) showed that with $40 \mathrm{~kg} / \mathrm{ha}$ of Trichoderma 
fertiliser increased yield in pepper was acquired. In our study, we applied $2.5 \mathrm{~kg} / \mathrm{ha}$ of VT and it might be insufficient quantity in winter and spring season. We can assume that low temperatures during few weeks in winter experiment, and occacional high temperatures above $30^{\circ} \mathrm{C}$ in spring trial, could negatively affect on microorganisms and their contribution to increased rossete fresh weight.

Rosette fresh weight was higher in spring and winter compared to autumn in all cultivars, which can be linked mostly to photoperiod length (autumn-short day 11-9 h, winter-growing day 9-13 h, spring-long day 14-15 h), and temperature, to a lesser extent. Optimal temperature for lettuce vegetative growth is $21-23^{\circ} \mathrm{C}$ (day) and $15-18{ }^{\circ} \mathrm{C}$ (night). Pavlou et al. (2007) reported decreased lettuce fresh weight in autumn, compared to spring and winter production, due to short day and sunshine duration.

Regarding to TAC, our results showed that red cultivars predominantly had a higher values compared to green, which is in accordance with the previous study (Llorach et al., 2008). The present results are in agreement with Gan and Azrina (2016), who reported the highest TAC in red coral lettuce (lollo rosso type). Along with cultivar 'Carmesi', higher antioxidant capacity also showed red cultivar 'Gaugin'. The lowest level was measured in cultivars 'Aquino' and 'Aleppo', both in spring. Application of all microbiological fertilisers led to increased TAC in cultivar 'Murai' in winter. Also, increased TAC showed cultivars 'Gaugin' and 'Aleppo' with fertilisers EMA, and combination of fertilisers, both in spring. Recent study on spinach pointed out the possibility to enhance TAC by using biofertiliser with beneficial fungi and rhizospheric bacteria (Khalid et al., 2017). Using of bacterial-algal preparation in romaine lettuce increased TAC 2.5 times in summer season, compared to control (Kopta et al., 2018). Along with genotype, environmental factors, like suboptimal conditions, can enhance TAC in order to impede oxidative stress in lettuce (Perez-Lopez et al., 2015). Application of microbiological fertilisers was season dependent in a way that lowered antioxidant capacity was noticed in autumn, with cultivar dependent response during spring and winter.

Phenolic content in lettuce is determined by genotype, agricultural/physiological conditions, and planting date (Liu et al., 2007). Sytar et al. (2018) showed that cultivar 'Carmesi' had the highest TPC among other red and green cultivars, almost under all environment conditions. In our study, the same cultivar showed the highest TPC in all treatments. TPC values of our samples were in the range of those found in lettuce (Oh et al., 2009; Gan and Azrina, 2016). Red cultivars that we examined showed higher TPC than green within the same type, but there is variation between different types of lettuce (Table 4).
Previous reports indicated higher TPC in red coloured lettuce, compared to green (Liu et al., 2007; Llorach et al., 2008). Our experiments showed the highest TPC and TAC in red leaf lettuce, which is in agreement with Liu et al. (2007). Expectedly, TPC is considered to have a great share in TAC activity of lettuce plants (Llorach et al., 2004). The present study indicated strong positive correlation between TAC and TPC (Fig.3, $\mathrm{r}=0.91^{*}$ ), which is in agreement with the results of Llorach et al. (2004) that showed high correlation irrespective of extraction method. Nicolle et al. (2004) reported that total phenolics had about $60 \%$ share in total antioxidant capacity in various lettuce cultivars.

Application of VT led to a higher TPC in cultivar 'Gaugin' in autumn, and all fertilisers in cultivar 'Murai' in winter. Literature data showed higher TPC content in lettuce using biofertiliser containing yeast extracts (Zlotek and Swieca, 2016). Association of lettuce with arbuscular mycorrhizal fungi increased phenolic content per plant in all cultivars (Baslam et al., 2011). On the contrary, association between Salvia officinalis and Glomus intraradices showed decreased content of TPC in plant leaves (Geneva et al., 2010). In our experiments, and when compared to control, microbiological fertilisers mainly decreased level of TPC in winter trial without response during spring.

As a part of the protection strategy, plants produce antioxidants in different environmental stress conditions. According to Oh et. al (2009) mild stresses (heat shock at $40{ }^{\circ} \mathrm{C}$ for 10 minutes and chilling stress at $4{ }^{\circ} \mathrm{C}$ for $24 \mathrm{~h}$ ) had an impact on increasing activity of key genes in synthesis of phenolics, vitamin $\mathrm{C}$ and $\mathrm{E}$, indicating that mild stress can enhance production of antioxidant compounds increasing TPC and TAC values. Their results showed absence of negative effect on lettuce weight and yield when plants were exposed to mild stresses. Among environmental factors, temperature and light had a great role on the phenolic content (Oh et al., 2009; Sytar et al., 2018). Relatively low temperatures (suboptimal) influenced on higher accumulation of anthocyanins through increased PAL activity, and they contributed to the accumulation of phenolic compounds (Boo et al., 2011).

Red cultivars showed higher total carotenoids content compared to green within same lettuce type, which is in agreement with Perez-Lopez et al. (2015). Genotype hads a great influence on total carotenoids in different lettuce types (Mou, 2009). Cultivar 'Carmesi' showed the highest total carotenoids content in all treatments in winter, commonly followed by cultivar 'Gaugin'. Positive effect of fertilisers application was observed with combination of fertilisers in 'Aleppo' and 'Murai', as well as VT in 'Aleppo'. There are poor literature data about the effect of beneficial microorganisms on total carotenoids content in lettuce. 
Results of inoculation with arbuscular mycorrhizal fungi in lettuce resulted in higher total carotenoids content (Baslam et al., 2011). Beside genotype/cultivar, environmental factors can influence on total carotenoids content. Light is a factor affecting total carotenoids content according to their role in plant protection from photooxidative stress, and as a part of plant defense mechanisms, they also contribute to TAC (Nicolle et al., 2004). This had been confirmed in our study as positive correlation between TAC and total carotenoids (Fig.3, $\mathrm{r}=0.64^{* *}$ ). According to Fallovo et al. (2009), season significantly affected total carotenoids content in lettuce during two growing periods.

Hipol and Dionisio-Sese (2014) indicated that red lettuce cultivar exposed to higher light intensity showed higher TAC and enzyme activity, compared to green varieties. Our results are in agreement with these findings, leading to the highest POD activity in red cultivars 'Carmesi' and 'Gaugin' detected in spring. Opposite to other antioxidant components, application of all fertilisers led to increased enzyme activity almost in all seasons. Kwak et al. (1996) suggested that POD activity can be enhanced as a result of different abiotic and biotic stresses. In Brassica juncea plants treated with Trichoderma harzianum, POD activity was significantly enhanced compared to non-inoculated plants under salinity stress (Ahmad et al., 2015). Additionally, Kheirizadeh Arough et al. (2016) showed that combining biofertilisers led to increased POD activity in Triticale sp., compared to control plants in salinity stress. Chon et al. (2012) demonstrated that POD activity in lettuce plants was lowest at $20^{\circ} / 13^{\circ} \mathrm{C}$ (day/night) temperature, and it was increasinged at higher temperatures, reaching the highest activity at $25^{\circ} \mathrm{C} / 20{ }^{\circ} \mathrm{C}$ (day/night) temperature. These experiments indicated that POD activity can be decreased at lower day/night temperatures.

Our climatic data showed that during autumn season air temperatures were mostly optimal (1-4 weeks after transplanting), and from the second half of vegetation period they were suboptimal leading to temperatures below $0{ }^{\circ} \mathrm{C}$ ten days before harvest. In winter season, two days after transplanting, plants were exposed to cold stress with the minimum temperature of $-16.6{ }^{\circ} \mathrm{C}$. Cold stress lasted for 56 days, in which plants were exposed to temperatures below $0{ }^{\circ} \mathrm{C}$, followed by optimal temperatures for about 15 days and afterwards, until harvest, they were exposed to temperatures above $30^{\circ} \mathrm{C}$, for several hours during day. In spring season, during the day plants were exposed to temperatures above $30{ }^{\circ} \mathrm{C}$, while night temperatures mostly stayed around optimal level. Average day/night temperatures in spring season were $26.3 / 15.3{ }^{\circ} \mathrm{C}$, which was similar to day/night temperatures that contributed to highest POD activity in lettuce experiment found by Chon et al. (2012). According to presented data, lower temperatures in winter, and partly in autumn season favorably influenced on accumulation of TPC, and increasing TAC, while higher temperatures in spring season contributed to higher POD activity, especially in red cultivars 'Carmesi' and 'Gaugin'.

Kredics et al. (2003) showed that soil microorganisms are mostly mesophilic and resistant to low temperatures. Optimum temperature for bacterial and fungial growth is between $25-30{ }^{\circ} \mathrm{C}$, whereas bacteria are more tolerant to higher temperatures, compared to low temperature tolerant fungi (Pietikainen et al., 2005). Temperatures below $0{ }^{\circ} \mathrm{C}$, and above $35{ }^{\circ} \mathrm{C}$, could negatively affect soil microorganisms. During winter and spring trials, we can assume that temperatures weren't favorable for soil microorganisms, and that they could have antagonistic effect, leading to decreased values of tested parameters. Also, the most of the winter and spring trial plants were exposed to suboptimal temperatures, and hence exposed to stress. Such a complex response indicates careful selection of cultivars and fertilizers in relation to the season, and necessity for further investigation of genotypemicroorganisms-environment interaction. Comparing effectiveness between tested fertilisers, we can emphasize VT and EMA+VT fertilizers, which principally contributed to higher quantity and quality parameters of lettuce. Presumably, integration of two fertilizers (EMA and VT) could acquire synergistic effect on examined parameters.

\section{CONCLUSION}

Our results showed the impact of genotype, microbiological fertilizers, and season on the rosette fresh weight, as well as components of antioxidant activity. From autumn to spring, rosette fresh weight increased in all cultivars, being the highest in non-treated plants. Generally, green cultivars showed higher rosette fresh weight, whereas red cultivars showed higher antioxidant capacity regarding metabolites as well as enzyme activity. Application of microbiological fertilizers was season dependent: in autumn mainly Vital Tricho and combination of fertilizers contributed to higher rosette fresh weight, while in winter and spring had none, or negative effect, respectively. The same treatments lowered antioxidant capacity in autumn, with cultivar dependent response during spring and winter. On the other hand, in all seasons specific POD activity was enhanced by fertilizers in almost all cultivars. Such a complex response indicates the need for careful selection of cultivars and fertilizers in relation to the season. Thus, lettuce production in spring led to the highest fresh weight regardless of the treatment, while winter production with combined fertilizers provided lettuce as a rich source of bioactive compounds for a healthy human diet. 


\section{ACKNOWLEDGEMENTS}

The authors appreciate the company Iceberg Salat Centar, Belgrade, Serbia, for technical support during experiments. Also, this work was supported by the Serbian Ministry of Education, Science and Technological Development under Contract No. 451-03-68/2020-14/200053.

\section{Authors' contributions}

Milica Stojanović: Conceptualization, Field and Laboratory Investigation, Methodology, Writing-Original Draft, Visualization, Resources. Vuk Maksimović: Laboratory Investigation, Methodology, Validation, Writing-Review and Editing, Resources. Dragosav Mutavdžić: Data analysis, Visualization. Ivana Petrović: Laboratory Investigation, Methodology. Zorica Jovanović: Methodology, Validation, Writing-Review and Editing, Resources. Slađana Savić: Conceptualization, Laboratory Investigation, Methodology, Writing-Review and Editing, Validation. Jelena Dragišić Maksimović: Laboratory Investigation, Methodology, Writing-Review and Editing, Validation, Resources.

\section{REFERENCES}

Ahmad, P., A. Hashem, E. F. Abd-Allah, A. A. Alqarawi, R. John, D. Egamberdieva and S. Gucel. 2015. Role of Trichoderma harzianum in mitigating $\mathrm{NaCl}$ stress in Indian mustard (Brassica juncea L.) through antioxidative defense system. Front. Plant Sci. 6: 868.

Babalola, O. O. 2010. Beneficial bacteria of agricultural importance. Biotechnol. Lett. 32: 1559-1570.

Bal, U. and S. Altintas. 2006. Application of the antagonistic fungus Trichoderma harzianum (TrichoFlow WP ${ }^{\mathrm{TM}}$ ) to root zone increases yield of bell peppers grown in soil. Biol. Agric. Hortic. 24: 149-163.

Barickman, T. C., W. L. Sublett, C. Miles, D. Crow and E. Scheenstra. 2018. Lettuce biomass accumulation and phytonutrient concentrations are influenced by genotype, $\mathrm{N}$ application rate and location. Horticulturae. 4: 12.

Baslam, M., I. Pascual, M. Sánchez-Díaz, J. Erro, J. M. García-Mina and N. Goicoechea. 2011. Improvement of nutritional quality of greenhouse-grown lettuce by arbuscular mycorrhizal fungi is conditioned by the source of phosphorus nutrition. J. Agric. Food Chem. 59: 11129-11140.

Boo, H. O., B. G. Heo, S. Gorinstein and S. U. Chon. 2011. Positive effects of temperature and growth conditions on enzymatic and antioxidant status in lettuce plants. Plant Sci. 181: 479-484.

Böhm, V., N. L. Puspitasari-Nienaber, M. G. Ferruzzi and S. J. Schwartz. 2002. Trolox equivalent antioxidant capacity of different geometrical isomers of $\alpha$-carotene, $\beta$-carotene, lycopene and zeaxanthin. J. Agric. Food Chem. 50: 221-226.

Bradford, M. M. 1976. A rapid and sensitive method for the quantitation of microgram quantities of protein utilizing the principle of protein-dye binding. Anal. Biochem. 72: 248-254.

Chon, S. U., H. O. Boo, B. G. Heo and S. Gorinstein. 2012. Anthocyanin content and the activities of polyphenol oxidase, peroxidase and phenylalanine ammonia-lyase in lettuce cultivars. Int. J. Food Sci. Nutr. 63: 45-48.
Cooperstone, J. L. and S. J. Schwartz. 2016. Recent insights into health benefits of carotenoids. In: Handbook on Natural Pigments in Food and Beverages. Woodhead Publishing, United States, pp. 473-497.

Dubova, L., I. Alsina and V. Steinberga. 2012. Comparison of Trichoderma spp. use efficiency on cucumbers and lettuce. Sci. Pap. Ser. B Hortic. 56: 101-104.

Dudaš, S., I. Šola, B. Sladonja, R. Erhatić, D. Ban and D. Poljuha. 2016. The effect of biostimulant and fertilizer on "low input" lettuce production. Acta Bot. Croat. 75: 253-259.

Fallovo, C., Y. Rouphael, E. Rea, A. Battistelli and G. Colla. 2009. Nutrient solution concentration and growing season affect yield and quality of Latuca sativa L. var. acephala in floating raft culture. J. Sci. Food Agric. 89: 1682-1689.

FAOSTAT. 2017. FAO Statistical Databases. Available from: http://www. fao.org/faostat/en/\#data/qc. [Last accessed on 2019 Apr 06].

Fiorentino, N., V. Ventorino, S. L. Woo, O. Pepe, A. de Rosa, L. Gioia, I. Romano, N. Lombardi, M. Napolitano, G. Colla and Y. Rouphael. 2018. Trichoderma-based biostimulants modulate rhizosphere microbial populations and improve $\mathrm{N}$ uptake efficiency, yield and nutritional quality of leafy vegetables. Front. Plant Sci. 9: 743.

Flohe, L. and F. Ursini. 2008. Peroxidase: A term of many meanings. Antioxid. Redox Signal. 10: 1485-1490.

Gan, Y. Z. and A. Azrina. 2016. Antioxidant properties of selected varieties of lettuce (Lactuca sativa L.) commercially available in Malaysia. IFRJ. 23: 2357-2362.

Geneva, M. P., I. V. Stancheva, M. M. Boychinova, N. H. Mincheva and P. A. Yonova. 2010. Effects of foliar fertilization and arbuscular mycorrhizal colonization on Salvia officinalis L. growth, antioxidant capacity, and essential oil composition. J. Sci. Food Agric. 90: 696-702.

Higa, T. and J. F. Parr. 1994. Beneficial and Effective Microorganisms for a Sustainable Agriculture and Environment. Vol. 1. International Nature Farming Research Center, Atami.

Hipol, R. L. B. and M. L. Dionisio-Sese. 2014. Impact of light variation on the antioxidant properties of red lettuce. Electron. J. Biol. 10: 28-34.

Khalid, M., D. Hassani, M. Bilal, F. Asad and D. Huang. 2017. Influence of bio-fertilizer containing beneficial fungi and rhizospheric bacteria on health promoting compounds and antioxidant activity of Spinacia oleracea L. Bot. Stud. 58: 35.

Kheirizadeh Arough, Y., R. Seyed Sharifi, M. Sedghi and M. Barmaki. 2016. Effect of zinc and bio fertilizers on antioxidant enzymes activity, chlorophyll content, soluble sugars and proline in Triticale under salinity condition. Not. Bot. Horti Agrobo. 44: 116-124.

Kim, M. J., Y. Moon, J. C. Tou, B. Mou and N. L. Waterland. 2016. Nutritional value, bioactive compounds and health benefits of lettuce (Lactuca sativa L.). Food Comp. Anal. 49: 19-34.

Kopta, T., M. Pavlikova, A. Sękara, R. Pokluda and B. Maršálek. 2018. Effect of bacterial-algal biostimulant on the yield and internal quality of lettuce (Lactuca sativa L.) produced for spring and summer crop. Not. Bot. Horti Agrobo. 46: 615-621.

Koudela, M. and K. Petríkova. 2008. Nutrients content and yield in selected cultivars of leaf lettuce (Lactuca sativa L. var. crispa). Hortic. Sci. 35: 99-106.

Kowalska, J. 2011. Effects of Trichoderma asperellum [T1] on Botrytis cinerea [Pers.: Fr.], growth and yield of organic strawberry. Acta Sci. Pol. Hortoru. 10: 107-114.

Kredics, L., Z. Antal, L. Manczinger, A. Szekeres, F. Kevei and E. Nagy. 2003. Trichoderma strains with biocontrol potential. Food Technol. Biotechnol. 41: 37-42. 
Kwak, S. S., S. K. Kim, I. H. Park and J. R. Liu. 1996. Enhancement of peroxidase activity by stressed-related chemicals in sweet potato. Phytochemistry. 43: 565-568.

Lichtenthaler, H. K. and A. R. Wellburn. 1983. Determinations of total carotenoids and chlorophylls $\mathrm{a}$ and $\mathrm{b}$ of leaf extracts in different solvents. Biochem. Soc. Trans. 11: 591-592.

Liu, X., S. Ardo, M. Bunning, J. Parry, K. Zhou, C. Stushnoff, F. Stoniker, L. Yu and P. Kendall. 2007. Total phenolic content and DPPH radical scavenging activity of lettuce (Lactuca sativa L.) grown in Colorado. LWT Food Sci. Technol. 40: 552-557.

Llorach, R., F. A. Tomas-Barberan and F. Ferreres. 2004. Lettuce and chicory byproducts as a source of antioxidant phenolic extracts. J. Agric. Food Chem. 52: 5109-5116.

Llorach, R., A. Martínez-Sánchez, F. A. Tomás-Barberán, M. I. Gil and F. Ferreres. 2008. Characterisation of polyphenols and antioxidant properties of five lettuce varieties and escarole. Food Chem. 108: 1028-1038.

Lopez-Bucio, J., R. Pelagio-Floresa and A. Herrera-Estrella. 2015. Trichoderma as biostimulant: Exploiting the multilevel properties of a plant beneficial fungus. Sci. Hortic. 196: 109-123.

Mampholo, B. M., M. M. Maboko, P. Soundy and D. Sivakumar. 2016. Phytochemicals and overall quality of leafy lettuce (Lactuca sativa L.) varieties grown in closed hydroponic system. J. Food Qual. 39: 805-815.

Miller, N. J., J. Sampson, L. P. Candeias, P. M. Bramley and C. A. Rice-Evans. 1996. Antioxidant activities of carotenes and xanthophylls. FEBS Lett. 384: 240-242.

Molla, A. H., M. M. Haque, M. A. Haque and G. N. M. Ilias. 2012. Trichoderma-enriched biofertilizer enhances production and nutritional quality of tomato (Lycopersicon esculentum Mill) and minimizes NPK fertilizer use. Agric. Res. 1: 265-272.

Mou, B. 2009. Nutrient content of lettuce and its improvement. Curr. Nutr. Food Sci. 5: 242-248.

Nicolle, C., A. Carnat, D. Fraisse, J. L. Lamaison, E. Rock, H. Michel, P. Amouroux and C. Remesy. 2004. Characterisation and variation of antioxidant micronutrients in lettuce (Lactuca sativa folium). J. Sci. Food Agric. 84: 2061-2069.

Oh, M. M., E. E. Carey and C. B. Rajashekar. 2009. Environmental stresses induce health promoting phytochemicals in lettuce. Plant Physiol. Biochem. 47: 578-583.

Ozcan, T., A. Akpinar-Bayizit, L. Yilmaz-Ersan and B. Delikanli. 2014. Phenolics in human health. IJCEA. 5: 393-396.

Pavlou, G. C., C. D. Ehaliotis and V. A. Kavvadias. 2007. Effect of organic and inorganic fertilizers applied during successive crop seasons on growth and nitrate accumulation in lettuce. Sci.
Hortic. 111: 319-325.

Pérez-López, U., J. Miranda-Apodaca, A. Muñoz-Rueda and A. MenaPetite. 2015. Interacting effects of high light and elevated $\mathrm{CO}_{2}$ on the nutraceutical quality of two differently pigmented Lactuca sativa cultivars (Blonde of Paris Batavia and Oak Leaf). Sci. Hortic. 191: 38-48.

Pérez-Urrestarazu, L., J. Lobillo-Eguíba, R. Fernández-Cañero and V. M. Fernández-Cabanás. 2019. Food safety concerns in urban aquaponic production: Nitrate contents in leafy vegetables. Urban For. Urban Green. 44: 126431.

Pietikainen, J., M. Pettersson and E. Baath. 2005. Comparison of temperature effects on soil respiration and bacterial and fungal growth rates. FEMS Microbiol. Ecol. 52: 49-58.

Priyadi, K., H. Abdul, T. H. Siagian, C. Nisa, A. Azizah, N. Raihani and K. Inubushi. 2005. Effect of soil type, applications of chicken manure and effective microorganisms on corn yield and microbial properties of acidic wetland soils in Indonesia. Soil Sci. Plant Nutr. 51: 689-691.

Sharma, P., A. N. Patel, M. K. Saini and S. Deep. 2012. Field demonstration of Trichoderma harzianum as a plant growth promoter in wheat (Triticum aestivum L). J. Agric. Sci. 4: 65.

Singleton, V. L. and J. A. Rossi. 1965. Colorimetry of total phenolics with phosphomolybdic-phosphotungstic acid reagents. Am. J. Enol. Vitic. 16: 144-158.

Stojanović, M., I. Petrović, M. Žuža, Z. Jovanović, Đ. Moravčević, G. Cvijanović and S. Savić. 2020. The productivity and quality of Lactuca sativa as influenced by microbiological fertilisers and seasonal conditions. Zemdirbyste. 107: 345-352.

Sytar, O., M. Zivcak, K. Bruckova, M. Brestic, I. Hemmerich, C. Rauh and I. Simko. 2018. Shift in accumulation of flavonoids and phenolic acids in lettuce attributable to changes in ultraviolet radiation and temperature. Sci. Hortic. 239: 193-204.

Tošić, I., Z. Golić and A. Radosavac. 2016. Effects of the application of biofertilizers on the microflora and yield of lettuce (Lactuca sativa L.). Acta Agric. Serbian. 21: 91-98.

Weather Underground. 2019. Available from: https://www. wunderground.com. [Last accessed on 2018 Dec 20].

Zahir, Z. A., A. Munir, H. N. Asghar, B. Shaharoona and M. Arshad 2008. Effectiveness of rhizobacteria containing ACC deaminase for growth promotion of peas (Pisum sativum) under drought conditions. J. Microbiol. Biotechnol. 18: 958-963.

Złotek, U. and M. Swieca. 2016. Elicitation effect of Saccharomyces cerevisiae yeast extract on main health-promoting compounds and antioxidant and anti-inflammatory potential of butter lettuce (Lactuca sativa L.). J. Sci. Food Agric. 96: 2565-2572.

Author Queries???

AQ1: Kindly cite figures 1 and 2 in the text part 